\title{
Rat muscle opacity decrease due to the osmosis of a simple mixture
}

\author{
Luís Oliveira, Armindo Lage, M. Pais Clemente, Valery V. Tuchin
}

\begin{abstract}
It is known that the fibrous structure of muscle causes light scattering. This phenomenon occurs due to the refractive index dis- continuities located between muscle fibers and interstitial fluid. To study the possibility of reducing light scattering inside muscle, we consider its spectral transmittance evolution during an immersion treatment with an optical clearing solution containing ethanol, glyc- erol, and distilled water. Our methodology consists of registering spectral transmittance of muscle samples while immersed in that so- lution. With the spectral data collected, we represent the transmit- tance evolution for some wavelengths during the treatment applied. Additionally, we study the variations that the treatment has caused on the samples regarding tissue refractive index and mass. By analyzing microscopic photographs of tissue cross section, we can also verify changes in the internal arrangement of muscle fibers caused by the immersion treatment. Due to a mathematical model that we develop, we can explain the variations observed in the studied parameters and estimate the amount of optical clearing agent that has diffused into the tissue samples during the immersion treatment. At the end of the study, we observe and explain the improvement in tissue spectral transmittance, which is approximately $65 \%$ after 20 min.
\end{abstract}

\section{Keywords}

Optical clearing; biomedical optics; refractive index matching; tissue transmittance; optical immersion.

\section{Introduction}

The study and development of optical techniques dedicated to perform clinical diagnosis and therapy has evolved rapidly in the last decade. However, the natural and strong scattering properties that biological tissues present limit the depth of light penetration. ${ }^{1}$ Different biological tissues exist in nature with particular optical properties. As an example, skeletal muscle is a fibrous tissue that presents strong light scattering that results in poor light penetration. ${ }^{2}$ The optical clearing technique enables reducing light scattering by immersing the tissues into optical clearing agents (OCAs) that have high refractive indices and hyperosmolarity. Such a technique enhances the capabilities of optical diagnosis and therapeutic treatments when combined with other approaches in optical methods. ${ }^{1}$

To obtain more valuable information from spectroscopic, confocal microscopy and optical coherence tomography (OCT), as well as from nonlinear spectroscopies such as two- photon fluorescence and second-harmonic generation (SHG) techniques, it is necessary to study and explain the mechanisms of optical clearing of fibrous tissues. ${ }^{3}$ SHG imaging microscopy, in particular, has recently been used to study and create images of skeletal muscle. The application of different concentrations of diluted glycerol as contrast agents has enables obtaining greater penetration depths in muscle samples. ${ }^{4}$ The combination of clearing mechanisms and SHG signal intensity measurements has made it possible to create 3-D images of muscle tissues. The usefulness of optical clearing in enhancing SHG images of muscle samples was observed and studied, showing that higher imaging depths are achieved. ${ }^{5}$

The human body contains large quantities of skeletal muscle. The principal function of this tissue is to provide the movement of bones and body parts. It is a very important tissue in human anatomy. The internal structure of the muscle consists on orientated muscle fibers surrounded by interstitial fluid (IF), which contains mainly water and some dissolved salts and minerals. ${ }^{2}$ Considering such internal composition and in the interest of developing and improving clinical optical technologies, it is important to know the optical properties 
of the muscle to optimize methods. Due to the heterogeneous composition of skeletal muscle, its refractive index profile presents ${ }^{2}$ localized discontinuities at the boundaries between the muscle fibers and the IF. This is due to the lower refractive index of water when compared to the refractive index of the muscle fibers. It is known ${ }^{6}$ that human muscular fibers have a refractive index of 1.41 , which is higher than the refractive index of IF distributed around the fibers. We know ${ }^{2}$ that for visible wavelengths, the main component of IF is water which has a refractive index of 1.33 .

Considering the tissue optics of the muscle, the refractive index mismatch between fibers and IF will cause major light scattering for an optical beam traveling inside. ${ }^{2}$ Such a phenomenon is evidence that the application of optical methods to perform diagnosis or treatment in the muscle will be limited. To optimize optical technologies in medicine, and in particular with application to the muscle, we can use a recent technique called optical immersion that creates refractive index matching and reduces light scattering. The process of refractive index matching is created by substituting the water in the IF by an optical clearing solution that diffuses into the interfiber space by osmosis. Such solutions have a refractive index higher than water. ${ }^{2}$ To develop a particular application with this method, it is necessary to understand the diffusion mechanism of the optical clearing solution into the tissue and the characteristics of the effect created. Probably the most important characteristics of the optical clearing effect are magnitude and time duration. Also, there are different types of tissues and different types of OCAs, each one a particular case, which means the necessity of extensive research.

One way to study the optical clearing effect created in biological tissues by the immersion technique is to study in vitro spectral transmission of tissue samples. This was the methodology used in our research. ${ }^{7}$ Our objectives consist of studying the optical clearing effect created in muscle samples by optical clearing solutions. With such study, we intend to obtain information to quantify optical transmittance increase of the tissues, identify the time dependence of the evolution of that increase, verify the refractive index matching that occurs inside the samples, and correlate the effect with histological variations that we have measured. We performed several studies with different OCAs applied as immersion liquids to muscle samples. Here, we present the study performed with a simple mixture containing ethanol, glycerol, and distilled water.

\section{Materials and Methods}

\subsection{Materials: Sample and Solution Preparation and Description}

To conduct our research we adopted abdominal wall muscle from the Wistar Hanrat. The rat skeletal muscle is very similar to human muscle in both function and composition. In this way, by using rat muscle, our study provides results and variations that can be considered closely approximated to a hypothetical study performed with human muscle samples. The muscle samples used in our study were dissected from the animal postmortem and then sliced with a cryostat into approximately uniform, 0.5 -mm-thick squares, approximately $1.5 \mathrm{~cm}$ on a side.

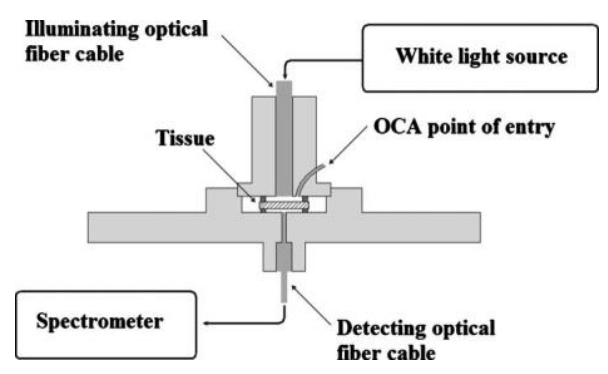

Fig. 1 Cross-section of tissue support chamber with illuminating and detecting optical fiber cables.

This study we used an optical clearing solution that we prepared as a simple mixture containing ethanol (density, $0.8 \mathrm{~g} / \mathrm{cm}^{3}$ ), glycerol (density, $1.26 \mathrm{~g} / \mathrm{cm}^{3}$ ), and distilled water (density, $1 \mathrm{~g} / \mathrm{cm}^{3}$ ) in the proportion of 1:1:2. We chose the mixture components after considering their potential in creating the optical clearing effect without arming or disrupting the tissue. As described in the literature, ${ }^{8}$ this type of solution was already used to create optical clearing effects in biological tissues. Ethanol provides biological membrane permeability and water reduces glycerol viscosity turning it easier to insert into the tissue. ${ }^{8}$ Some authors ${ }^{9}$ have also studied the influence of alcohols on the optical clearing processes, verifying that they are more efficient, and glycerol, in particular, with three hydroxyl groups has better efficiency than those with one of two groups.

\subsection{Methods}

Since our study includes different measurement techniques and methods, we describe each one here independently.

\subsubsection{Transmittance measurement}

Before and after each transmittance study, we measured the tissue mass to identify mass variations caused by the treatment applied with the OCA. To perform these measurements we used a PM2000 weighing scale manufactured by Mettler with a precision of $0.01 \mathrm{~g}$. In all the mass measurements, the tissue samples were carefully dried with absorbing paper to eliminate water or OCA from their surface.

The study of the optical clearing effect was performed by measuring and representing the temporal evolution of the transmittance spectra of the muscle samples when immersed in the solution. To measure spectral transmittance, we constructed a special chamber with black nylon to contain the muscle samples and prevent the detection of parasite radiation. the tissue support system and chamber are represented in cross section. Figure 1 presents the samples were placed inside a chamber that has the optical fiber cable from the light source above it and the optical fiber cable to the spectrometer below. The light source was a xenon white light lamp and the spectrometer was an Ocean Optics s2000 spectrometer with a spectral acquisition band from 200 to $1100 \mathrm{~nm}$. The sample was illuminated continuously and the transmittance measurements were registered by the spectrometer and stored in a file. All the processes of spectral registration were performed with OOIBase software from Ocean Optics. The transmittance 
measurements were made for five muscle samples during the experiment, and the results presented represent the mean of these five experimental studies.

For each muscle sample, we initiated the transmittance measurements by placing the sample inside the chamber between the optical fiber cables. We then measured its natural transmittance, and after that initiated the immersion treatment by dropping the OCA from a lateral opening of the chamber. The volume of OCA used in each experimental study was twice the volume of the tissue samples $(2 \times 1.5 \times 1.5$ $X 0.05 \mathrm{~cm}=0.225 \mathrm{~cm}^{3}$ ). Considering this volume, we can calculate the mass of OCA used in each experiment

$$
\begin{aligned}
M_{\mathrm{OCA}}= & \left(V_{\mathrm{OCA}}\right)(\text { combined density })=(0.789+1.261+1 * 2) \\
& \mathrm{X}(0.225)=0.911 \mathrm{~g} .
\end{aligned}
$$

The liquid spread around the tissue sample almost immediately immersing it completely, and we began transmittance measurements at the same instant. Measurements were taken every $5 \mathrm{~s}$ during the first $2 \mathrm{~min}$. After 2 min of tissue immersion, we took measurements every $30 \mathrm{~s}$ for the next $6 \mathrm{~min}$ and every minute after that. Due to the lack of significant changes seen on the computer screen in spectral transmittance after $20 \mathrm{~min}$, we decided to interrupt the study.

\subsubsection{Refractive index measurement}

To complement the transmittance evolution study performed on the muscle samples during optical immersion, we also measured the refractive index values of natural and treated samples to analyze variations. We used an Abbe refractometer with red contrast illumination to perform the refractive index measurements. The samples used in these measurements were not the same ones used in the transmittance measurements, since we could not obtain accurate measurements of refractive index from samples with thicknesses of approximately $0.5 \mathrm{~mm}$ due to their high turbidity. We therefore prepared other samples of abdominal wall muscle, but now with an approximately uniform thickness of $0.4 \mathrm{~mm}$. Several samples were prepared, in both natural and treated states, to be used in the refractive index measurement procedure. The refractometer used was an Ar4 model from A. Krüss Optronic (Germany). From the samples were cleaned with soft absorbing paper to make them dry on the outside before measuring the refractive index. Then the samples were placed in the refractometer sensing area, over the prism surface. This was done with care to guarantee that the whole sample was uniformly laid over the prism. The upper arm of the refractometer was closed over the sample and a red diode laser was placed to illuminate the sample. Such sample illumination results in better contrast when reading the refractive index from the refractometer scale through the ocular. Although the contrast illumination was at $633 \mathrm{~nm}$, the sample refractive index readings were referred to at $589 \mathrm{~nm}^{2}$

\subsubsection{Histological parameter evaluation of tissues}

The internal structure changes caused by the treatment were also identified by comparing microscopic photographs taken from natural and treated samples. Natural and treated samples were submitted to a long preparation process to be photographed with the microscope. The first step of tissue prepara- tion consisted of placing the tissue samples, suspended between two microscope glasses, in a bath of Bouin's fixative liquid for $12 \mathrm{~h}$. The purpose was to fix the cells inside the tissue and harden it for further processing. After this period, the samples are submitted to a sequence of immersion procedures in different concentrations of alcohol to obtain complete dehydration. The procedure consists of three immersions in $70 \%$ alcohol solutions for $15 \mathrm{~min}$ each, then an other three immersions in $90 \%$ alcohol for $10 \mathrm{~min}$ each, and finally, three more immersions in $100 \%$ alcohol for $10 \mathrm{~min}$ each. Next, the tissue samples were completely dehydrated and placed in a benzyl solution to fill the tissue with paraffin it for to maintain posterior slicing procedures. The tissue was immersed in the benzyl solution twice, for $5 \mathrm{~min}$ each. We then obtained a block of paraffin containing the tissue inside. These blocks were then sliced to obtain cross-sectional films of the tissue used in the transmittance study. The tissue films were then placed in xylol solution for 10 min to clear the paraffin substrate, then in $100 \%$ alcohol for another $10 \mathrm{~min}$, and finally in $90 \%$ alcohol for another $10 \mathrm{~min}$. The tissue samples were then placed in water for $15 \mathrm{~min}$ before beginning the dyeing process, which began by immersing tissue-paraffin films in hematein for $2 \mathrm{~min}$ to dye the cell nuclei. Later, they are washed in running water for $15 \mathrm{~min}$ to remove the color residues that were dispersed from the paraffin film. Finally, each sample was immersed for $30 \mathrm{~s}$ in $1 \%$ eosin to color the cytoplasm in each tissue cross section. This process spread the dye coloring in the support that sustains the paraffin with tissue inside. We then washed the coloring dye from the paraffin film outside the tissue using successive washes in $90 \%$ alcohol. Later the tissue films were washed in absolute alcohol and finally in xylol until the paraffin films were completely transparent. The tissue films were then placed inside two thin microscope glasses for protection. Such glasses are fixed to each other with varnish and some time is required for the varnish to dry. At the end, the microscope glasses containing tissue cross sections are in condition to be observed and photographed at the microscope.

The microscope enables observation and photographing with several orders of magnitude. However, for our study it was sufficient to obtain photographs with $2 \mathrm{X}$ and $10 \mathrm{X}$ magnification. Photographs of a length scale were also taken with the same magnitudes to perform the measurements.

For each of the five natural and five treated samples, we took 10 thickness measurements along the $2 \mathrm{X}$ photographs. To accomplish this, we placed a transparency print of the length scale over the print of each tissue photograph. Then we considered the mean thickness of the five natural tissues and the mean thickness of the five treated tissues. All the thickness measurements made for each tissue are very similar and they are not too different from the mean values calculated.

\section{Results}

As already described, our experimental study consisted of the application of different techniques to obtain different kinds of information. The first step consisted of characterizing the muscle in terms of volume fractions for solid and liquid parts and to estimate the corresponding refractive indices. This process is described in Sec. 3.1. Section 3.2 presents the results obtained in the transmittance study during optical immersion 
of tissue. Finally, in Sec. 3.3 we present histological photographs, for both natural and treated samples, to study the variations caused by the optical treatment.

\subsection{Natural Muscle Characterization}

To estimate the refractive indices and volume fractions of muscle constituents, we used a suitable theoretical model that we have developed based on the measurements obtained with the Abbe refractometer and a weight scale during a study of sample dehydration with a hairdryer. ${ }^{10}$ The collected pairs of mass and refractive index measurements corresponding to the sequential dehydration states enabled us to establish a correspondence between dehydration and the volume fractions of water and the solid part inside the muscle, as well as a dependence between the refractive index and sample weight. Considering the natural state of the tissue before dehydration was applied, we can use the Gladstone and Dale law, presented as Eq. (2), to describe the measured refractive index of the natural muscle sample as the sum of the weighted portions of each constituent: ${ }^{2}$

$$
n_{\text {natural }}=f_{\text {water }} n_{\text {water }}+f_{\text {solid part }} n_{\text {solid part }} .
$$

The mean refractive index measured from five natural samples was 1.3980. The volume fraction of water in natural muscle is described in the literature ${ }^{11}$ as 0.756 . The refractive index of water is 1.3330 according to our calculations. ${ }^{10} \mathrm{By}$ considering a total volume of $100 \%$ for the muscle sample, we can establish a relation between the volume fractions of tissue constituents:

$$
f_{\text {water }}+f_{\text {solid part }}=1 \text {. }
$$

Using the volume fraction of water already presented, we can use Eq. (3) to obtain the volume fraction of the solid part of the muscle as 0.244 . Now, using the two volume fractions and the refractive indices of water and measured from the natural muscle sample, we can use Eq. (2) to estimate the refractive index of the solid part. This estimation is not precise if we do not consider the mechanisms of dehydration occuring in the tissue. When considering such mechanisms, ${ }^{10}$ we obtain the refractive index for the solid part of rat muscle as 1.584 . This refractive index value is higher than the refractive index that we measured directly from the natural sample, since it was obtained from the data collected from a dehydrated tissue, with more dense packing of the mucle fibers. ${ }^{10}$ All the previous values characterize the natural muscle in terms of volume fractions and refractive indices for the corresponding constituents.

The next step was to measure the refractive index of the optical clearing solution that we have used in the study. Since the solution is very translucent, only one refractive index measurement was performed. This value is presented in Table 1 .

\subsection{Spectral Transmittance Study}

After acquiring all the necessary refractive index measurements from the tissue sample and OCA, we initiated our spectral transmittance study. The study was performed on five muscle samples, so we could calculate mean spectra for better accuracy in the representation of the temporal evolution of
Table 1 Mean values of mass and refractive index for natural and treated tissue samples and OCA.

\begin{tabular}{lcc}
\hline Mean Values & Mass $(\mathrm{g})$ & Refractive Index \\
\hline Natural tissue samples & $0.14 \pm 0.01$ & $1.3980 \pm 0.00005$ \\
Treated tissue samples & $0.11 \pm 0.01$ & $1.3855 \pm 0.00005$ \\
OCA (E:G:W=1:1:2) & 0.911 & $1.3805 \pm 0.00005$ \\
Mean variations in tissue & -0.03 & -0.0125 \\
\hline
\end{tabular}

tissue transmittance. From these five natural samples, we also measured mass. The mean mass of the natural samples used in the study is also presented in Table 1 along with the mean refractive index measured from another five natural samples. For each sample, we began the optical clearing transmit- tance study by placing the sample inside the central dark chamber.

We then measured the natural transmittance spectrum of the sample. Due to great internal scattering, the natural muscle presents a low transmittance, as we can verify from Fig. 2. Figure 2 shows the mean natural transmittance spectrum of rat muscle, which is a typical transmittance for a fibrous tissue. The transmittance rises with wavelength. Due to the thourough washing out of blood from the samples before spectral analysis, in Fig. 2 we cannot see the hemoglobin Soret band (415 to $420 \mathrm{~nm}$ ) and the $Q$-bands (530 to $575 \mathrm{~nm}$ ).

Then we dropped the OCA into the chamber to immerse the sample, and began to take transmittance measurements. We next present some evolution graphs for tissue transmittance. We verified that the osmotic diffusion of the solution into the tissue was very fast at the beginning, saturating the tissue almost immediately, as we can see in Fig. 3 .

The time evolution curves presented in Fig. 3 represent the mean spectral values from the five studies for some welldistributed wavelengths that are indicated in the figure. In this figure, the time resolution is $1 \mathrm{~min}$ and all the represented

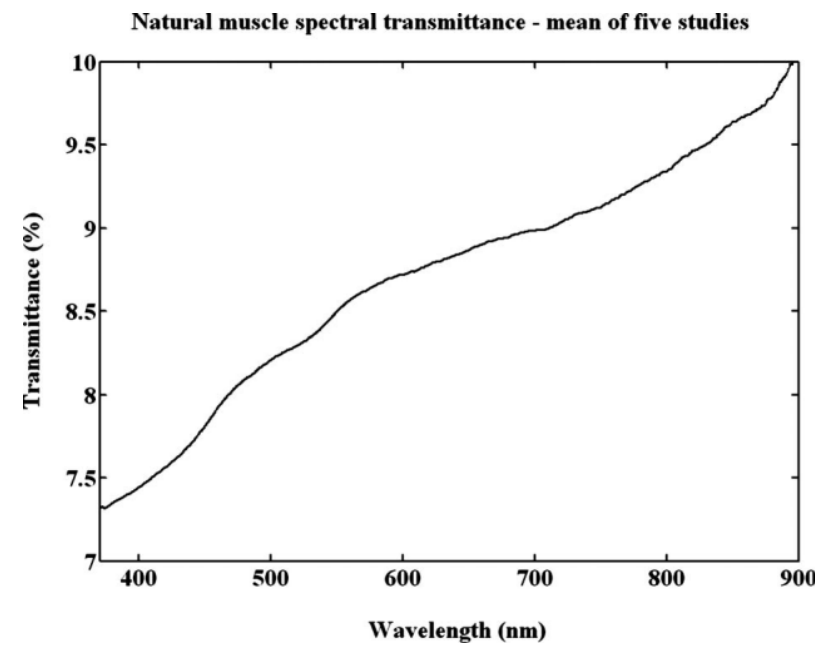

Fig. 2 Mean natural transmittance of rat muscle (the mean thickness of samples was $0.551 \mathrm{~mm}$ ). 


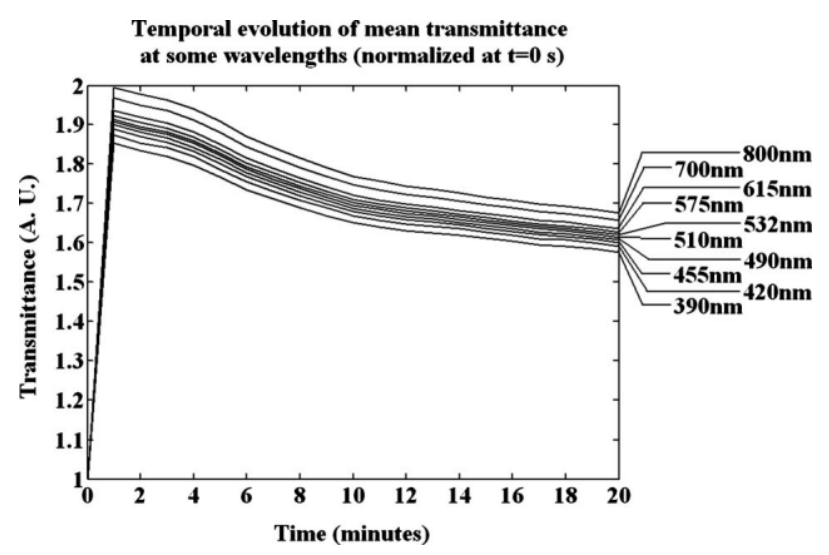

Fig. 3 Normalized temporal evolution of the mean transmittance of the tissues during treatment with the optical clearing solution.

lines corresponding to the different wavelengths were normalized to the correspondent natural transmittance, for a better understanding of the rapid increase that occurs in the first minute of optical immersion. As we can see from Fig. 3, transmittance almost doubles for all wavelengths selected after $1 \mathrm{~min}$ of treatment. The twofold increase observed in the mean transmittance curves of Fig. 3 indicates that in average the absolute transmittance with wavelength dependence, as represented in Fig. 2, will increase from 7.5 to 10 to 15 to $20 \%$. In the following minutes transmittance presents a slowly decreasing behavior, which tends to stabilize after $10 \mathrm{~min}$. Also, we verify that even after 20 min of optical immersion, transmittance presents values around $65 \%$ higher than in the natural state.

With the objective to better understand the strong initial increase in spectral transmittance, we represent in Fig. 4(a) the evolution of transmittance in the first 2 min with a 5-s resolution. Such a representation was made for the same wavelengths used in Fig. 3. This representation also has the objective to verify whether there are any oscillations in the transmittance evolution, as we verified in studies with other optical clearing solutions. ${ }^{7,12}$

Fig. 4(b) presented the temporal evolution of the mean transmittance spectrum for the tissues studied with the solution. In this representation, we verify the occurrence of absorption bands in the spectra measured at 5, 10, 15, 20, and $25 \mathrm{~s}$ of the applied osmotic treatment. This phenomenon was also observed in the studies that we performed ${ }^{7,12}$ with other OCAs. These absorption bands become more evident due to the normalization applied to the spectra that eliminates the natural spectral form of tissue transmittance.

Figure 4(a) shows that the mean transmittance at specific wavelengths always presents a growing tendency during the first $30 \mathrm{~s}$ and it inverts this tendency very smoothly after that. There are no significant oscillations such as were verified in other cases. ${ }^{7,12}$ On the other hand, from Fig. 4(b), we can see that in fact the entire mean tissue transmittance spectrum shows a well-defined growing tendency in the first $30 \mathrm{~s}$.

At the end of the spectral studies, we measured mass again from the treated tissues to determine the variations caused by the application of the immersion treatment. The mean values calculated for the five experimental studies are presented in

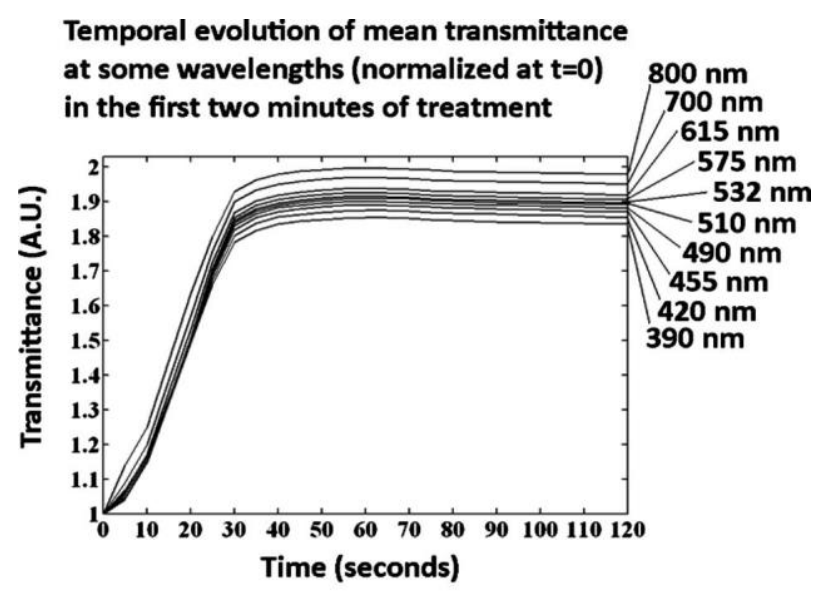

(a)

Temporal evolution of mean transmittance spectrum of muscle immersed in the mixture in the first two minutes of treatment

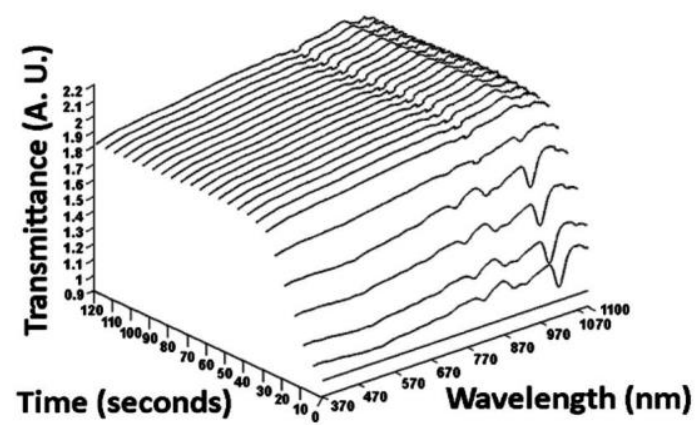

(b)

Fig. 4 Mean transmittance evolution of tissues treated with the mixture in the first $2 \mathrm{~min}$ for (a) some wavelengths and (b) the whole spectrum.

Table 1. Comparing the data from natural and treated samples in Table 1, we verify that the treatment with the optical clearing solution caused a decrease in mean tissue mass of $0.03 \mathrm{~g}$ and a decrease of the global refractive index from 1.3980 to 1.3855 .

\subsection{Histological Variations Analysis}

Finally, we used microscope photographs to study the internal geometrical variations that the treatment produced by the exchange between water and the optical clearing solution. To do this, we consider the photographs presented in Fig. 5, which correspond to natural and treated states of the rat muscle. The photographs presented in Figs. 5(a) and 5(b) were taken by the microscope with $2 \mathrm{X}$ magnification and correspond to natural tissue and treated tissue samples, respectively. Figures 5(c) and 5(d) show the 10X magnification photos of the same tissue samples for comparison. Figure $5(\mathrm{c})$ is the magnification of the frame in Fig. 5(a) and Fig. 5(d) is a magnification of the frame in Fig. 5(b).

From the photographs in Figs. 5(a) and 5(b) we made thickness measurements to detect thickness variations caused by the applied treatment. The mean thickness determined for 


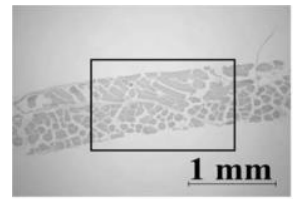

(a)

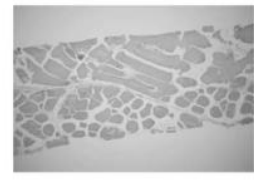

$0.5 \mathrm{~mm}$

(c) (b)

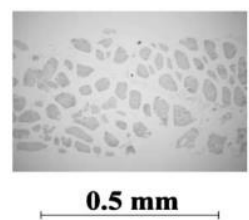

(d)

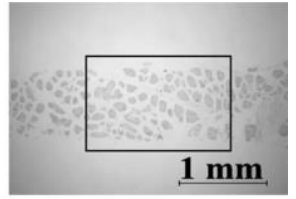

Fig. 5 Cross-section photographs of natural and treated tissues with different microscope magnifications: (a) $2 X$ natural tissue, (b) $2 X$ treated tissue, (c) 10X natural tissue, and (d) 10X treated tissue.

the five natural samples was $0.551 \mathrm{~mm}$, and for the treated samples it was $0.816 \mathrm{~mm}$.

\section{Discussion}

From Figs. 3 and 4 we verify that the optical immersion of the tissues in the mixture results in an increase of the mean transmittance for a long period of time. All the curves represented in Figs. 3 and 4(a) show a similar behavior. In particular, from these two figures, we verify that the curves show higher transmittance values for higher wavelengths throughout the time period of treatment, which is certainly related with the form

of the natural transmittance spectrum of the muscle that rises

with wavelength, as represented in Fig. 2. This natural char-

acteristic of the muscle favors applications that use higher wavelengths such as surgical or therapeutic applications, whose wavelengths are located between $600 \mathrm{~nm}$ and the IR. Observing Fig. 4(a), the lack of significant oscillations at the initial $10 \mathrm{~s}$ of the immersion treatment is probably due to the presence of water in the optical clearing solution, which makes the fluid exchange very smooth and uniform at the beginning

of the process. This fact indicates that solutions containing water exchange with the IF in an orderly manner - a fact to be considered for future solutions to develop.

Due to the spectral normalization that we performed in Fig. 4(b), the natural shape of the transmittance spectrum was eliminated. This fact enables us to better identify the absorption bands that were created during the initial growing process. Considering the wavelength scale, the first two absorption bands present a small intensity, but the last is quite accentuated. Since these bands are visible only for measurements performed in the first $25 \mathrm{~s}$, they are certainly related with water flow inside the tissues. Spectral oscillations between 970 and $1070 \mathrm{~nm}$ could be associated with water band at $980 \mathrm{~nm}$, because for this wavelength, scattering is not very high and the absorption bands could be seen. However, the scattering strength is enough to make absorption band easily visible due to the multiplicity of chaotic photon migration paths, which increase the probability of photons being absorbed. In contrast, at optical clearing when scattering goes down, the absorption bands arrive with less contrast because the overall probability of a photon being absorbed has also gone down. For all cases studied with different OCAs, it is amazing how the absorption bands occur in the transmittance spectrum at the same time instants and enable us to identify the period of time in which the dehydration flows occur.

Considering the variations presented in Table 1, from the natural to the treated samples, the mass loss is caused by the water loss from the tissue. The decrease in the refractive index of the tissues is caused by the refractive index matching that occurs due to the dehydration of the tissue and the diffusion of OCA into the interfiber spaces inside the tissue and its better optical homogeneity. A comparison of Figs. 5(c) and 5(d) shows that the insertion of OCA into the tissue forces the muscle fiber bundles to separate. Also, due to the presence of water in the OCA used, the tissue does not dehydrate strongly. As a consequence of this separation between the muscle bundles and the low dehydration of the tissue, scattering is reduced and the refractive index reduction might be due to this lower scattering of the sample. With this variation in refractive index presented in Table 1 and considering Eq. (2), we can apply that equation to the treated state of the sample to estimate the volume fraction that has been inserted into the tissue. For the treated state of the sample, we can write

$$
n_{\text {treated }}=f_{\mathrm{OCA}} n_{\mathrm{OCA}}+\left(1-f_{\mathrm{OCA}}\right) n_{\text {natural }} .
$$

Now, solving Eq. (4) in order of $f_{\mathrm{OCA}}$, we have

$$
f_{\text {OCA }}=\frac{n_{\text {treated }}-n_{\text {natural }}}{n_{\text {OCA }}-n_{\text {natural }}} .
$$

Using this equation and solving it with the measured refractive indices, we obtain a value of 0.714 . Comparing this value with the volume fraction of water inside the natural tissue (0.77), we verify that after the treatment, the volume percentage of agent inside is slightly less than the volume percentage of water in the natural state of the tissue. However, we should consider that the agent itself contains $50 \%$ distilled water in its composition, and this way, the amount of water inside the treated tissue must be of 0.357 . This water content in the treated tissue is less than half the water content in the natural sample. This means that water has been reduced greatly inside the tissue due to the treatment applied.

To interpret the mass variation, we have performed some calculations to verify sample mass variation with the applied treatment. In these calculations, we describe sample mass as the sum of the water and solid part masses, each one calculated as the product of the corresponding volume per its density. We describe the natural sample mass as

$$
M_{\text {natural }}=p_{\text {solid }} V_{\text {solid }}+p_{\text {water }} V_{\text {water }}
$$

For the natural state of the muscle, we know that the water volume is approximately 0.77 and its density is $1.00 \mathrm{~g} / \mathrm{cm}^{3}$. On the other hand, the solid part occupies a volume of 0.23 and has an unknown density. We can substitute water volume fraction and density to simplify Eq. (6):

$$
M_{\text {natural }}=p_{\text {solid }} V_{\text {solid }}+1 \times 0.77 .
$$

If we suppose that all the optical clearing mixture that we have estimated to enter the tissue sample has replaced tissue 
water, the treated tissue has the same solid part (equal volume and density), 0.714 of agent and some remains of water $(0.77-0.714=0.056)$. The agent inside is composed of $25 \%$ glycerol, $25 \%$ ethanol, and $50 \%$ water. With these percentages in the agent, we see that glycerol and ethanol occupy 0.1785 of volume each (25\% of 0.714$)$ and water occupies 0.357 (50\% of 0.714 ). We also know the corresponding densities of ethanol and glycerol. Glycerol has a density of $1.26 \mathrm{~g} / \mathrm{cm}^{3}$, and ethanol a density of $0.8 \mathrm{~g} / \mathrm{cm}^{3}$. The water total volume in the treated tissue is $0.413(0.056+0.357)$ and its density is the same as in natural tissue. With these data we can write mass for treated tissue as

$$
\begin{aligned}
M_{\text {treated }}= & p_{\text {solid }} V_{\text {solid }}+p_{\text {water }} V_{\text {water }}+p_{\text {glycerol }} V_{\text {glycerol }} \\
& +p_{\text {ethanol }} V_{\text {ethanol }}
\end{aligned}
$$

If we replace volumes and densities for water, glycerol, and ethanol, we can rewrite Eq. (8) as a function of only solid part density and volume:

$$
M_{\text {treated }}=p_{\text {solid }} V_{\text {solid }}+1 \times 0.413+1.26 \times 0.1785+0.8
$$

$$
\mathrm{X} 0.1785 \text {. }
$$

All the arithmetic operations in Eq. (9) can be made to obtain treated mass as a function of only solid part mass and volume:

$$
M_{\text {treated }}=p_{\text {solid }} V_{\text {solid }}+0.7807 \text {. }
$$

Now we can calculate mass difference by subtracting the natural mass to the treated mass:

$$
M_{\text {treated }}-M_{\text {natural }}=\left(p_{\text {solid }} V_{\text {solid }}+0.7807\right)-\left(p_{\text {solid }} V_{\text {solid }}+0.77\right) \text {. }
$$

If we simplify the common terms in Eq. (11), we obtain the difference of masses:

$$
M_{\text {treated }}-M_{\text {natural }}=0.7807-0.77=0.0107 \mathrm{~g} \text {. }
$$

This mass difference suggests that the treatment has caused a small weight increase of $0.0107 \mathrm{~g}$ and not a weight loss of $0.3 \mathrm{~g}$, as measurements indicated.

This result may not correspond to the reality of the fluid exchange during the immersion treatment. It is well known that glycerol accumulates water. ${ }^{13}$ In this way, supposing that glycerol does not enter tissue in large amounts, but that ethanol does, and each molecule of water flux from tissue will be bounded by glycerol placed on the sample surface, i.e., this water portion left the tissue forever, we get to about 0 for the term in parenthesis:

$$
\begin{aligned}
M_{\text {treated }}= & p_{\text {solid }} V_{\text {solid }}+p_{\text {water }} V_{\text {water }}+\left(p_{\text {glycerol }} V_{\text {glycerol }}\right) \\
& +p_{\text {ethanol }} V_{\text {ethanol }} .
\end{aligned}
$$

As a maximum of this rough estimation, the mass of treated tissue presented in Eq. (10) will be less on $0.2142 \mathrm{~g}$, because $p_{\text {glycerol }} V_{\text {glycerol }}=1.26 X 0.1785=0.2249 \mathrm{~g}$.

This value is not far from the measured value of $0.3 \mathrm{~g}$, however, in reality a more complex situation is possible: because ethanol also accumulates water (since it is a dehydration agent), some amount of glycerol may enter into tissue and any glycerol molecule outside tissue may accumulate more than one water molecule. According to Ref. 13, each molecule of glycerol attracts up to about six molecules of water. In addition, glycerol can diffuse into tissue and may accumulate water in tissue as well; however, its outside concentration is much higher than within tissue sample. Thus, water flux leaving tissue will be accumulated by outside glycerol and dehydration will occur.

Finally, we can conclude that balance between these multifluxes of water, glycerol, and ethanol will lead to tissue dehydration and loss of weight.

To explain the decrease observed in the mean refractive index caused by the optical clearing we now present and describe the optical model of inhomogeneous media. The refractive index $n$ of the inhomogeneous medium is described as ${ }^{14}$

$$
n_{\text {tissue }}=\bar{n}+\frac{\overline{n^{2}}-\bar{n}^{2}}{\bar{n}} Q(\lambda / l),
$$

where

$$
\bar{n}=n_{0}\left[1+\left(\frac{n_{s}}{n_{0}}-1\right) \frac{V_{s}}{V_{0}}\right],
$$

where $V_{s}$ is the total volume of scattering particles; $V_{0}$ is the volume of the scattering medium; $n_{0}$ and $n_{s}$ are refraction coefficients of the background material and inserted scatterers, respectively; $n^{2}$ is the mean-square value of refractive index fluctuations; the $Q(\mathrm{X} / l)$ function refers to the form of scatterers and their aggregation; $l$ is the correlation length of a randomly distributed refractive index fluctuation. Note that $Q=1.17$ in the limit of large correlation length $l$ (large particles) and $Q=0$ in the limit of small $l$ (Rayleigh limit). In the case that the refractive index fluctuations take the form of parallel cylinders, which is the case of muscle fibers, $Q$

$=0.67$ for the large $l$.

More concentrated (ordered) muscle fibers are associated with a smaller mean-square value of refractive index fluctuation, when $n^{2}-\bar{n}^{2}$ and the mean value of tissue refractive index $n_{\text {tissue }}$ should drop to an $\bar{n}$ value defined by a weighted mixture of two types of media-scatter and background material. In our experiments, we found a lesser mean refractive index for more cleared and less inhomogeneous, i.e., less scattering muscle tissue (see Table 1).

Finally, we interpret the histological variations observed in the photographs presented in Fig. 5. Muscle tissue is mainly composed of muscle cells (muscle fibers) containing myofibrils, which are composed of thin (actin) and thick (myosin) filaments. Muscle fibers are bound together into bundles called fascicles (well seen in Fig. 5). Accounting for muscle structure and comparing between Figs. 5(a) and 5(b) we can evaluate the global changes caused by the treatment. These changes were significant in terms of geometrical distribution of the fiber bundles and correspondingly of fiber density. We can see that the treatment applied to the tissues caused the muscle fibers to separate from each other, leaving emptier areas in between. Evidently, the major scatterers of the tissue, which are muscle fibers/myofibrils/filaments (packed into bundles, i.e., fascicles), became more densely packed. Moreover, the areas between the muscle fiber bundles that were previously occupied by the IF, are now more or less saturated with OCA. Looking at the $10 \mathrm{X}$ photographs presented in 
Figs. 5(c) and 5(d) we can see more exactly relative changes in spaces occupied by fiber bundles and IF. In Fig. 5(c), we can see that the natural muscle fiber bundles are surrounded by considerable quantities of IF. The muscle bundles present different cross sections because some of them were obliquely sliced. This means that they do not have exactly the same orientation. When we look at the treated tissue presented in Fig. 5(d), we see that the space between the muscle fiber bundles is greater than in the case of the natural tissue. Both effects, more dense packing of dehydrated muscle fibers (cells) and increased refractive index of IF in the space between fiber bundles, make the tissue less scattering and therefore more optically transparent. A related phenomenon was found by investigating the optical clearing at application of $50 \%$ glycerol solution on skeletal mouse muscle tissue by the second-harmonic microscopy. ${ }^{4}$ The authors of Ref. 4 showed experimentally on intact tissue that the transparency of skeletal muscle tissue was substantially increased ( -2.5 -fold). In this paper, it was also stated that immersing muscle in glycerol does not significantly change the chemical and structural properties of the muscle contractile system. However, such a treatment permeabilizes the fiber cellular membrane, and thus extracts a fraction of the cytoplasmic proteins, which makes system less scattering.

Based on our measurements and histology we can hypothesize that not only proteins but also water and other cellular components may leave cells at increased cell membrane permeation and being collected in the space between fiber bundles. Thus, fibers are more densely packed within bundles [see low area of bundles at clearing in Fig. 5(d)] and the refractive index of space between fiber bundles could be greater than the initial value. Glycerol diffusion into this space, as already mentioned, also helps to increase refractive index of the IF.

We cannot say for sure that the sample thickness increase we observed was caused by optical clearing treatment that we applied. In fact, such a thickness increase is contradictory with observed loss of mass. One possible cause for this thickness increase is the methodology used for tissue preparation to obtain histology photographs at the microscope. In such a methodology (see Sec. 2.2.3), we have inserted the tissue samples inside two microscope glasses for long periods of time. It is possible that due to this applied stress, the treated tissue expanded after being released from the microscope glasses. It has been shown that cardiac muscle changes its mechanical properties, becoming more rigid after OCA treatment. ${ }^{15}$ For our muscle tissue, such information is not known, but it is possible that it also changes its mechanical properties with OCA treatment, leading to the thickness increase that we observed. The possibility of this change in the mechanical properties of the tissue should be further investigated in the future.

\section{Conclusions}

Considering the results obtained with our research, we can characterize the transparency effect created by this solution in the muscle. When immersed in the solution, the muscle almost doubled its natural transmittance in the first $30 \mathrm{~s}$ and this value tended to lower after that time period. This fast initial upgrade in optical transmittance was caused by the presence of glycerol in the optical clearing solution. In the subsequent instants of the treatment, water assumed the most important role since it represents $50 \%$ of the solution. The optical clearing process is not naturally reversible in in vitro samples and tends to maintain a high transmittance for a long period of time, as we observed in Fig. 3. For the mean spectral evolution that we considered, the muscle transmittance after 20 min was approximately $65 \%$ higher than in the natural state. These variations are evidence of tissue dehydration and agent diffusion into the interfiber spaces inside the tissue. The decrease verified in the global refractive index of the tissue explains the phenomenon of refractive index matching that occurred in the interfibers space. The internal redistribution of the muscle fibers inside the tissue, as well as the visualized decrease in fiber diameters, demonstrated the insertion of agent into the tissue and the creation of an osmotic pressure on the muscule fibers. On the other hand, we must account for the high proportion of water in the solution used. This water will partially substitute for water that left the tissue at the beginning due to the stimulated dehydration.

Note the usefulness of this agent in clinical applications in which it is necessary to quickly create a transparency effect and in cases where only a short time for that effect is necessary. Some diagnostic applications, such as for instance, the fact that photodiagnosis can benefit from the creation of a transparency effect of this type. Considering the results obtained, we think that preparing similar solutions with different concentrations of water, glycerol, and ethanol might produce interesting results and different characteristics for the optical clearing effect created. This is a case to be studied in the near future.

\section{Acknowledgments}

The authors are thankful to Professor Deolinda Lima Teixeira and the Institute of Histology and Embryology, Professor Abel Salazar, for all the support given to accomplish the presented work. VVT is thankful for support by Grants 224014 Photonics4life-FP7-ICT-2007-2; RUB1-2932-SR-08 CRDF; RF Ministry of Science and Education Grants 2.1.1/4989, 2.2.1.1/2950, and 1.4.09; RFBR-08-02-92224-NNSF_a (RFChina); RFBR-10-02-90039-Bel_a; and RF governmental contracts $\quad 02.740 .11 .0484, \quad 02.740 .11 .0770, \quad$ and 02.740.11.0879.

\section{References}

1. X. Wen, Z. Mao, Z. Han, V. V. Tuchin, and D. Zhu, "In vivo skin optical clearing by glycerol solutions: mechanism," J. Biophoton. 3(1-2), 44-52 (2010).

2. V. V. Tuchin, Optical Clearing of Tissues and Blood, SPIE Optical Engineering Press, Bellingham, WA (2006).

3. V. V. Tuchin, "A clear vision for laser diagnostics (review)," IEEE J. Sel. Top. Quantum Electron. 13(6), 1621-1628 (2007).

4. S. Plotnikov, V. Juneja, A. Isaacson, W. Mohler, and P. Campagnola, "Optical clearing for improved contrast in second harmonic generation imaging of skeletal muscle," Biophys. J. 90(1), 328-339 (2006).

5. R. laComb, O. Nadiarnykh, S. Carey, and P. Campagnola, "Quantitative second harmonic generation imaging and modeling of the optical clearing mechanism in striated muscle and tendon," J. Biomed. Opt. 13(2), 21109-21111 (2008).

6. J. Mobley and T. Vo-Dinh, "Optical properties of tissue," Chap. 2 in Biomedical Photonics Handbook, T. Vo-Dinh, Ed., pp. 2.1-2.75, CRC Press LLC, Boca Raton, FL (2003). 
7. L. M. Oliveira, Study of the Spectral Transmission Response of Biological Tissues Under the Influence of Different Osmotic Agents, FEUP Edições, Porto, Portugal (2007).

8. E. A. Genina, A. N. Bashkatov, Y. P. Sinichkin, V. I. Kochubey, N. A Lakodina, G. B. Altshuler, and V. V. Tuchin, "In vitro and in vivo study of dye diffusion into the human skin and hair follicles," $J$. Biomed. Opt. 7, 471-477 (2002).

9. Z. Mao, D. Zhu, Y. Hu, X. Wen, and Z. Han, "Influence of alcohols on the optical clearing effect of skin in vitro," J. Biomed. Opt. 13(2), 021104-021109 (2008).

10. L. M. Oliveira, A. S. Lage, M. A. Pais Clemente, and V. V. Tuchin, "Optical characterization and composition of abdominal wall muscle from rat," Opt. Lasers Eng. 47(6), 667-672 (2009).

11. R. F. Reinoso, B. A. Telfer, and M. Rowland, "Tissue water content in rats measured by desiccation," J. Pharmacol. Toxicol. Methods 38, 87-92 (1997).
12. L. M. Oliveira, A. S. Lage, M. A. Pais Clemente, and V. V. Tuchin, "Concentration dependence of the optical clearing effect created in muscle immersed in glycerol and ethylene glycol," Proc. SPIE 6535, 653511 (2007)

13. J. W. Wiechers, J. C. Dederen, and A. V. Rawlings, "Moisturizing mechanisms: internal occlusion by orthorhombic lipid phase stabilizers - a novel mechanism of action of skin moisturization," Chap. 19 in Skin Moisturization, 2nd ed., A. V. Rawlings and J. J. Layden, Eds., pp. 309-322, Informa Healthcare, New York (2009).

14. R. P. Hemenger, "Refractive index changes in the ocular lens result from increased light scatter," J. Biomed. Opt. 1, 268-272 (1996).

15. P. B. Wells, A. T. Yeh, and J. D. Humphrey "Influence of glycerol on the mechanical reversibility and thermal damage susceptibility of collagenous tissues," IEEE Trans. Biomed. Eng. 53, 747-753 (2006). 